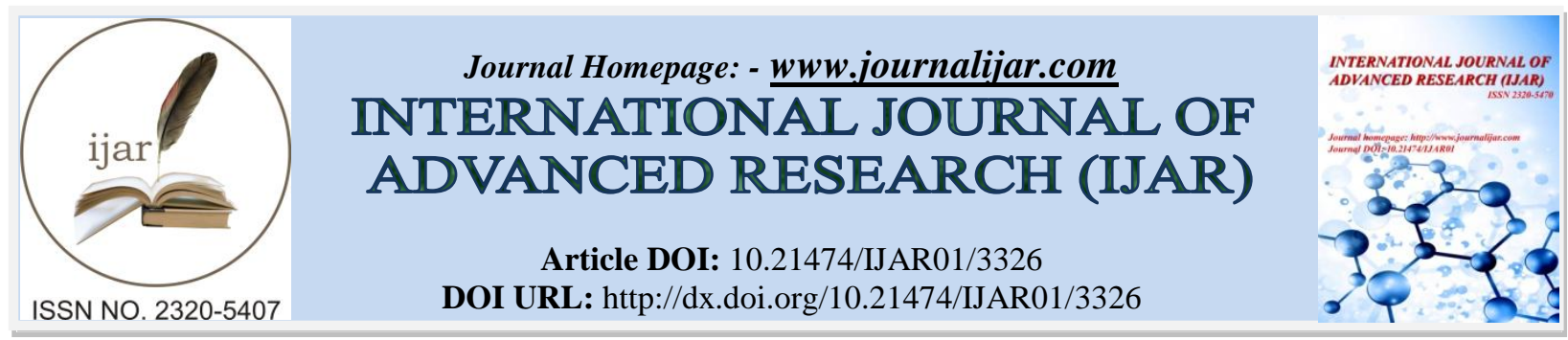

RESEARCH ARTICLE

\title{
EVALUATION OF BITE FORCE IN COMPLETELY AND PARTIALLY EDENTULOUS PATIENTS (PRE AND POST REHABILITATION.
}

Dr. Amrita Patnaik ${ }^{1}$, Dr.N. V. V. Satyabhushan ${ }^{2}$, Dr. U. Sivakalyan ${ }^{3}$ and Dr. Kho Chai Chiang ${ }^{4}$.

1. Postgraduate student, Department of Oral and Maxillofacial Surgery, GITAM Dental College and Hospital,Visakhapatnam.

2. Professor and Head, Department of Oral and Maxillofacial Surgery, GITAM Dental College and Hospital, Visakhapatnam.

3. Reader, Department of Oral and Maxillofacial Surgery, GITAM Dental College and Hospital, Visakhapatnam.

4. Senior Lecturer, Department of Oral and Maxillofacial Surgery, GITAM Dental College and Hospital, Visakhapatnam.

\section{Manuscript Info}

Manuscript History

Received: 23 December 2016

Final Accepted: 22 January 2017

Published: February 2017

Key words:-

Maximum Bite Force, Pre-Rehabilitation

Bite Force, Post Rehabilitation Bite

Forces,Edentulous,Dentulous.

\begin{abstract}
The aim of this study was to determine the maximum bite force prior and post rehabilitation in completely and partially edentulous patients rehabilitated with either complete denture, removable partial denture, fixed partial denture or implants in comparison with the control group in 75 patients belonging to each group who were randomly selected after clinical examination. A total of 75 patients made up the study population. The study was carried out with the aim of measuring the maximum bite force using the bite force meter. Comparison was made amongst the bite forces of control group with that of the study groups. Implants followed by fixed partial denture group exhibited an excellent increase in pre to post rehabilitation stages with its values evenly distributed between the genders across all the study groups. Further, a greater bite force was exhibited in fully dentate patients (controls) in the overall study. Higher bite forces were elicited in patients rehabilitated with implants and is considered as the most effective mode of rehabilitation with maximum bite force in comparison with the other rehabilitation methods.
\end{abstract}

Copy Right, IJAR, 2017,. All rights reserved.

\section{Introduction:-}

Mastication is a highly coordinated neuromuscular function involving purposeful movements of the jaw and continuous modulation of force, representing a function that matures with growth as the teeth erupts. It is characterized by complex movements of the stomatognathic system structures, most importantly the mandible, which varies depending on the foods ingested, resulting in manipulation of a food bolus, salivation, and associated oscillatory movements of the head. The loss of teeth, and consequently, prosthodontic rehabilitation poses new challenges to the stomatognathic system, which alters masticatory function. Relatively little is known about the extent to which chewing differs between dentate subjects and completely or partially edentulous patients treated in various ways. ${ }^{6,14,15,19,28,41}$.

Corresponding Author:- Dr. Amrita Patnaik.

Address:- Postgraduate student, Department of Oral and Maxillofacial Surgery, GITAM Dental College 
Edentulism is defined as the loss of permanent teeth and is believed to be caused by dental caries, periodontal disease, trauma and others. It is an indicator of both population health and the functioning and adequacy of a country's oral health care system and is associated with low education level and poor socioeconomic status. ${ }^{1,2}$.

The advances in the medical sciences have led to people living longer. Along with the increased life expectancy, there is a desire to live to the fullest with an esthetic appearance and functional rehabilitation. The patients who have lost their teeth are looking for custom made treatment modality in prosthetic rehabilitation. This helps in providing adequate masticatory, phonetic and esthetic function, simulating the natural dentition that does not jeopardize the remaining teeth, ${ }^{41}$ thereby ranging from a complete coverage single crown or a long span fixed dental prosthesis to a full mouth rehabilitation. ${ }^{5 .}$ Loss of teeth can be equated to an amputation, and gaps in the teeth are perceived as physical imperfections akin to missing body parts. ${ }^{9}$.

Adult patients with varying severity of tooth loss can be rehabilitated by different types of fixed or removable constructions which are retained by the remaining natural teeth or dental implants, or in cases of edentulism, are supported directly by the oral mucosa.

At present, there exists three main methods which are used individually or in combination in order to replace lost teeth. The three methods are as follow:

1. A removable denture, which the patient can insert or remove.

2. A bridge retained by the natural teeth abutting the gap and which bears the replacement teeth.

3. Implants which are anchored in the jawbone and support a removable denture or fixed tooth-replacements.

Bite force is an important element of masticatory system. ${ }^{23}$. The bite force is the ratio of the distance from the jaw joint which is the fulcrum or pivot point to the point of application, i.e. the biting versus the distance from the jaw joint to the muscle attachment which is the force required to close the jaw. This force results from the action of the jaw elevator muscles(in turn ,determined by the central nervous system and feedback from muscle spindles, mechanoreceptors and nociceptors) modified by the craniomandibular biomechanics.

Masticatory performance is a cumulative contribution of various factors like bite force, severity of malocclusion, occlusal contact area, body loss of teeth, restorations, facial forms and other motor activities. Maximum bite force indicates the functional state of the masticatory system. ${ }^{7}$ Bite forces are correlated to diet, cranial design and are considered to be the key determinant of the masticatory function.

Factors including bite force and occlusal contact area, suggest that higher the bite forces and the larger the occlusal areas, the more efficient the mastication is. Bite force has also shown to be affected by a number of physiological and morphological variables such as craniofacial morphology, age, gender, periodontal support of the teeth, height and body weight, tempro-mandibular disorders pain, and dental status. ${ }^{29}$.

Other variables reportedly affecting the bite force are the type of recording devices, technique employed to measure the bite force, position of the sensor in the oral cavity, patient position, unilateral or bilateral measurements and magnitude of mouth opening during measurements. ${ }^{4 .}$

\section{Patients and Methods:-}

After obtaining the ethical clearance, subjects for the present study were selected amongst the patients who reported to the outpatient department of Oral and Maxillofacial Surgery, GITAM Dental College and Hospital, Visakhapatnam from June 2014 to March 2015 to evaluate the pre and post rehabilitation bite forces in completely and partially edentulous patients.

Details of the patients were recorded at the beginning. Bite forces prior and post rehabilitation were recorded. All selected patients were informed about the experimental nature of the study. Their cooperation was solicited. The effective mode of rehabilitation was determined on the basis of pre and post rehabilitation bite forces. 


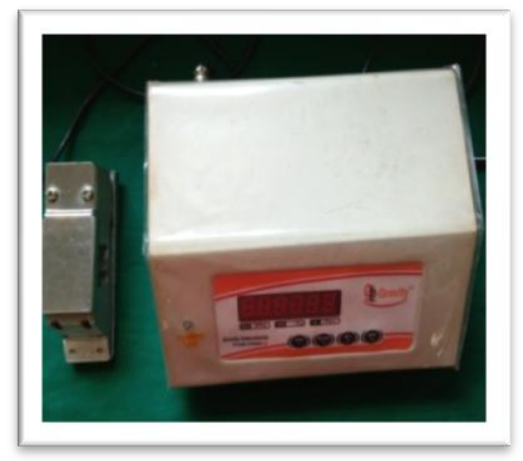

Bite force measurements were made using indigenous Bite Force Meter in 75 individuals belonging to different age groups. Follow up was done post rehabilitation. The maximum bite forces were assessed in the control group. Pre and Post rehabilitation bite forces were recorded in the study group which comprises complete denture, removable partial denture, fixed partial denture and implants.

The bite force meter consisted of a Wheatstone bridge assembly, instrument amplifier and a digital panel meter. An adjustable button is incorporated on the instrument for resetting the instrument reading to zero, at the start of each recording. This electronic device was connected to the bite force pads. The bite force pads were placed in the posterior $1^{\text {st }}$ molar region on both right and left sides of the jaw. This was accomplished by instructing the subject to bite on the pads as the subjects were asked to remain seated with the head upright, looking forward , and in an unsupported natural head position throughout the trial refraining from extraneous movements. The force applied is seen on the bite force meter display. The high precision load cell and electronic circuit provided precise measurements. ${ }^{10 .}$

\section{Results:-}

In the present study, the most common age groups that the subjects underwent rehabilitation procedures were between $35-53$ years. The mean age group was 43 years.

There was a significant increase in the post rehabilitation bite forces in males and females on both right and left side when compared to the pre rehabilitation bite forces in all the groups. However, the group comprising removable partial denture indicated a significant decline in the post rehabilitation bite forces in comparison to pre rehabilitation bite forces.

\begin{tabular}{|c|c|c|c|c|c|c|}
\hline \multicolumn{7}{|l|}{ Paired Samples Statistics } \\
\hline \multicolumn{3}{|l|}{ Group } & Mean & $\mathrm{N}$ & $\begin{array}{l}\text { Std. } \\
\text { Deviation }\end{array}$ & p-value \\
\hline \multirow[t]{4}{*}{ complete denture } & \multirow[t]{2}{*}{ Pair 1} & right & 10.8140 & 15 & 5.78130 & \multirow[t]{2}{*}{0.001} \\
\hline & & rightaftr & 22.1247 & 15 & 10.57687 & \\
\hline & \multirow[t]{2}{*}{ Pair 2} & Left & 12.3260 & 15 & 6.62137 & \multirow[t]{2}{*}{0.003} \\
\hline & & leftaftr & 23.9547 & 15 & 11.77417 & \\
\hline \multirow[t]{4}{*}{ removable partial denture } & \multirow[t]{2}{*}{ Pair 1} & right & 216.8453 & 15 & 289.18050 & \multirow[t]{2}{*}{0.269} \\
\hline & & rightaftr & 130.1687 & 15 & 36.83493 & \\
\hline & \multirow[t]{2}{*}{ Pair 2} & Left & 196.6040 & 15 & 253.47638 & \multirow[t]{2}{*}{0.908} \\
\hline & & leftaftr & 185.5500 & 15 & 221.86138 & \\
\hline \multirow[t]{4}{*}{ fixed partial denture } & \multirow[t]{2}{*}{ Pair 1} & right & 105.8340 & 15 & 30.51702 & \multirow[t]{2}{*}{0.000} \\
\hline & & rightaftr & 142.3220 & 15 & 29.76522 & \\
\hline & \multirow[t]{2}{*}{ Pair 2} & Left & 99.1333 & 15 & 32.25323 & \multirow[t]{2}{*}{0.000} \\
\hline & & leftaftr & 142.9213 & 15 & 35.02141 & \\
\hline \multirow[t]{4}{*}{ Implants } & \multirow[t]{2}{*}{ Pair 1} & right & 263.4800 & 15 & 44.21017 & \multirow[t]{2}{*}{0.000} \\
\hline & & rightaftr & 296.7827 & 15 & 45.55500 & \\
\hline & \multirow[t]{2}{*}{ Pair 2} & Left & 263.2633 & 15 & 42.46300 & \multirow[t]{2}{*}{0.000} \\
\hline & & leftaftr & 293.0387 & 15 & 43.53010 & \\
\hline
\end{tabular}

Higher bite forces were recorded from patients who were rehabilitated by fixed partial denture whereas the bite forces from the patients who were rehabilitated with Implants were found to be the highest. 


\section{Discussion:-}

As a result of rehabilitation of tooth loss, the patient's oral function gets restored and eventually the patient regains the prerequisite conditions for participating in social activities again. ${ }^{1,2}$. Specific improvements are described such as enjoyment of food, clarity of speech and attractive facial expression. Single tooth loss can be treated by a toothretained bridge, a resin-bonded retained bridge or an implant-retained crown. Few studies have shown that the survival of implant-retained crown constructions is over 90 percent and the risk of bone loss ( $>2 \mathrm{~mm})$ around the implant is small. Patients with more extensive tooth loss can be treated with tooth or implant-supported bridges. Around 95 percent of implant-supported bridges can be expected to survive after 5-10 years. ${ }^{5}$

Patients with long periods of edentia often lose more mandibular bone than those with a shorter period of edentia. Denture wearing is also very important in alveolar ridge resorption as the number of lower dentures worn is related with the period of edentia and alveolar ridge resorption. The individuals who wear their complete dentures continuously throughout the day and night, have more resorptive changes in the jaws when compared to those who wear dentures daily. The high success rate and consequently the widespread use of dental implants for prosthetic rehabilitation have led to revision of numerous aspects of the original treatment protocols. ${ }^{3 .}$ The aim of this report was to evaluate the patients livelihood with varying degrees of tooth loss and their response to the type of rehabilitation with the perseverance oral quality of life that the currently available methods of rehabilitation have after 5,10 and 15 years according to its efficiency.

Maximum bite force is a useful indicator of the functional state of the masticatory system and the loading of the teeth, and its recordings can be performed in a relatively simple way in the clinic or the department. ${ }^{22}$. A measuring device, essentially a bite force meter, is directed to a particular location, a bite point source of a specific tooth among the whole dentition and the bite force is measured. ${ }^{10 .}$

Comparison of bite forces between males and females on both right and left sides was done in the control group. The highest bite force was recorded on left side of females which was around $398 \mathrm{~N}$. The left side of control group individuals was more significant when compared with the other groups. The right and left posterior regions did not show any significant difference in bite force. The mean bite force was around $350 \mathrm{~N}$ on the right and $364 \mathrm{~N}$ on the left side of the jaw. Few studies have shown that increase in the maximum bite force values was seen at the dentate side when compared to the rehabilitated side. ${ }^{8}$. There may be hormonal differences that contribute to the composition of muscle fibers in both males and females. However the age factor has got relatively a small influence on the bite force. ${ }^{4}$.

Comparison of pre and post rehabilitation bite forces on right and left side of the jaw was done in all the study groups that comprised of complete denture, removable partial denture, fixed partial denture, and implants group.

In complete denture group, the pre rehabilitation bite forces was around $10 \mathrm{~N}$ on right side and $12 \mathrm{~N}$ on the left side that showed no statistical significant increase whereas post rehabilitation bite forces was around $22 \mathrm{~N}$ on right side and $23 \mathrm{~N}$ on left side that showed a statistical significant increase on the left side of the jaw when compared across all other groups. Hence, many studies have stated that, the stomatognathic system behaves functionally depending on the type of oral rehabilitation. 5 .

In removable partial denture group, there was a mild difference between the pre and post rehabilitation bite forces in the study group. Pre rehabilitation bite forces was around $216 \mathrm{~N}$ on right side and $196 \mathrm{~N}$ on left side where as post rehabilitation bite forces was around $130 \mathrm{~N}$ on right side and $185 \mathrm{~N}$ on left side. Post rehabilitation, bite forces were higher on the left side of females. Although studies on the influence of gender on maximum bite force have shown that in young adults, the females showed greater values when compared to males. Patients with mandibular anterior or posterior natural teeth have greater resorption of the edentulous maxilla than patients with mandibular complete dentures or patients with natural teeth. Hence studies have concluded that the cause of resorption of maxillary and mandibular bony structures was due to removable partial denture and mostly seen in removable partial denture wearers. ${ }^{3 .}$

In fixed partial denture group, the pre rehabilitation bite forces was around $105 \mathrm{~N}$ on right side and $99 \mathrm{~N}$ on left side where as post rehabilitation bite forces was around $142 \mathrm{~N}$ on both left and right side as well. Thus, no statistical significance was elicited in both the pre and post rehabilitation stage. Higher bite force values were recorded in females on the right side post rehabilitation. It is believed that female adults between 13 and 20 years of age exhibit 
a greater muscle thickness ${ }^{19}$ whereas males have a gradual increase in muscle thickness that eventually occurs over time. ${ }^{39}$. Many studies have proven that the occlusal force would be higher on the preferred chewing side and no statistical significance of occlusal force was found in relation to the quadrants. ${ }^{15}$. As masticatory performance was mainly affected by occlusion factor the greater areas of contact and near contact provided better occlusal stability (tooth alignment and molar bite force) which helped in allowing the masticatory function to be more efficient. ${ }^{27}$

In the implant group, the pre rehabilitation bite forces was around $263 \mathrm{~N}$ on right side and left side as well, whereas the post rehabilitation bite forces was around $296 \mathrm{~N}$ on the right side and $293 \mathrm{~N}$ on left side. The pre as well as post rehabilitation stages exhibited an excellent increase in the bite force when compared across all the groups. A statistically significant difference was seen between males \& females post rehabilitation on the left side for implant group. On comparing across the other rehabilitatory groups, higher bite force values were elicited in the implant group individuals. Several studies have concluded that, higher bite force and masticatory efficiency was seen in individuals rehabilitated with implants and single crowns. An improved function was seen in those individuals rehabilitated with implant supported mandibular over denture when compared to those rehabilitated with conventional complete dentures. ${ }^{5,20 .}$ The biting efficiency was affected by the postoperative occlusal plane angle. ${ }^{23 .}$ A positive correlation was found between muscle function and tooth position in few studies. ${ }^{28}$. The biting force measured for the ipsilateral and the contralateral pair of teeth has always shown higher values in the posterior pair of teeth. ${ }^{41}$ A minimum period of twelve weeks is required for the tissues to adapt to a new vertical dimension as the bite force measurements were found to give consistent readings after twelve weeks. ${ }^{22}$. A High score of satisfaction and quality of life has been found in patients rehabilitated with implant supported prosthesis. ${ }^{5,13 .}$

\section{Conclusion:-}

Implants exhibited the maximum bite force in comparison with all the other groups, due to which it stands as one of the most successful and effective mode of rehabilitation. Thus it can be advised that tooth or teeth loss can be rehabilitated with either implant or implant based treatment options as it achieves the same bite force as the natural teeth.

\section{References:-}

1. Subhash Sonkesariya, Deshraj Jain, Prabha Shakya, Rohit Agrawal, S V Sai Prasad - Prevalence of Dentulism, Partial Edentulism, and Complete Edentulism in Rural and Urban Population of Malwa Region of India: A Population based study, International Journal of Prosthodontics and Restorative Dentistry, October-December 2014, 4(4):112-119.

2. Karl Peltzer, Sandra Hewlett, Alfred E. Yawson, Paula Moynihan, Raman Preet, Fan Wu, Godfrey Guo , Perianayagam Arokiasamy, James J. Snodgrass, Somnath Chatterji, Mark E. Engelstad and Paul Kowal Prevalence of Loss of All Teeth (Edentulism) and Associated Factors in Older Adults in China, Ghana, India, Mexico, Russia and South Africa, International Journal of Environmental Research and Public Health, 2014, 11:11308-11324.

3. Dubravka Knezovie-Zlatarie, Asja elebie, Biserka Lazie- Resorptive Changes of Maxillary and Mandibular Bone Structures in Removable Denture Wearers, Acta Stomatol Croat, 2002,36( 2):261-265.

4. Duygu Koc, Arife Dogan, Bulent Bek, Bite Force and Influential Factors on Bite Force Measurements: A Literature Review, European Journal of Dentistry, April 2010, 4:223-232.

5. Laner B.Rosa, Cesar Bataglion, Selma Siessere, Marcelo Palinkas, Wilson Mestriner Junior, Osvaldo de Freitas, Moara de Rossi, Ligia Franco de Oliveira, Simone C.H. Regalo-Bite force and masticatory efficiency in individuals with different oral rehabilitations, Journal of Stomatology,2012, (2):21-26.

6. Julien Luraschi, Martin Schimmel, Jean-Pierre Bernard, German O.Gallucci, Urs Belser, Frauke MullerMechanosensation and maximum bite force in edentulous patients rehabilitated with bimaxillary implant supported fixed dental prostheses, Clinical Oral Implants Research, 2012, 23(5):577-583.

7. Duygu KOC, Arife DOGAN, Bulent BGK-Effect of gender, facial dimensions, body mass index and type of functional occlusion on bite force, Journal of Applied Oral Sciences,2011,19(3):274-279.

8. Bader K.Al-Zarea-Maximum Bite Force following Unilateral Fixed Prosthetic Treatment: A Within -Subject Comparison to the Dentate side, Medical Principles and Practice, 2014, 24:142-146.

9. Suzana Varga, Stjepan Spalj, Marina Lapter Varga, Sandra Anic Milosevic, Senka Mestrovic, Mladen SlajMaximum voluntary molar bite force in subjects with normal occlusion, European Journal of Orthodontics,2011,33:427-433. 
10. Veena Jain, Vijay Prakash Mathur, Rajath Sasidharan Pillai, Sandeep Kalra-A preliminary study to find out maximum occlusal bite force in Indian individuals, Indian Journal of Dental Research,2014,25(3).

11. Mansour Rismanchian, Farshad Bajoghli, Zahra Mosta-Effect of Implants on Maximum Bite Force in Edentulous patients, Journal of Oral Implantology,2009,15(4).

12. Hakan Bilhan, Onur Geckili, Emre Mumcu, Altug Cilingir, Ergen Bozdag-The influence of implant number and attachment type on Maximum bite force of mandibular overdentures, Gerodontology, 2012, 29:116-120.

13. Onur Geckili, Hakan Bilhan, Emre Mumcu, Necat Tuncer-The influence of Maximum Bite Force on Patient Satisfaction and Quality of Life of Patients Wearing Mandibular Implant Overdentures ,Journal of Oral Implantology,2012,38(3).

14. Gaurav Tripathi, Ponnana A.A, Nishant Rajwadha, Nidhi Chhaparia, Abhishek Sharma, Mahendra AnantComparative Evaluation of Maximum Bite Force in Dentulous and Edentulous Individuals with Different Facial Forms,Journal of Clinical and Diagnostic Research,2014 September,8(9):ZC37-ZC40.

15. TeutaBicaj,Teuta Pustina, Enis Ahmedi, Linda Dula, Zana Lila, Arlinda Tmava -Dragusha, Resmije AdemiAbdyli, Nexhmije Ajeti-The relation between the preferred Chewing Side and Occlusal Force Measured by TScan III System, Journal of stomatology,2015,5:95-101.

16. Patricia Takaki, Marena Vieira, Silvana Bommarito- Maximum Bite Force Analysis in Different Age Groups, International Archives of Otolaryngology,2014,18(3).

17. Bedoya. A, Osorio, J.C, Tamayo, J.A- Dental Arch Size, Biting Force, Bizygomatic Width and Face Height in Three Colombian Ethnic Groups, International Journal of Morphology,2015,33(1):55-61.

18. Carolyn M. Eng, Daniel E. Lieberman, Katherine D. Zink, Michael A. Peters-Bite Force and Occlusal Stress Production In Hominion Evolution, American Journal of Physical Anthropology,2013,151:544-557.

19. Frauke Muller, Marta Hernandez, Linda Grutter, Luis Aracil-Kessler, Dieter Weingart, Martin SchimmelMasseter muscle thickness, chewing efficiency and bite force in edentulous patients with fixed and removable implant-supported prostheses: a cross sectional multicenter study, Clinical Oral Implantology,2012,23:144-150.

20. Raghunath Patil, Jayashree Sajjanar, Mahantesh Bembalagi, Pavithra kumar Shetty-Bite Force in Tooth Supported Prostheses, Journal of Dental and Medical Sciences, January 2015,14(1):66-68.

21. Katsuyoshi Sakaguchi, Sawako Uehara, Takakazu Yagi, Shouichi Miyawaki-Relationship between occlusal curvatures and bite force in humans, Journal of Orthodontic Waves, 2012, 71:79-84.

22. Urbano Santana-Mora, Arturo Martinez-Insua, Urbano Santana-Penin, Amaya P.del Palomar, Jose C.Banzo, Maria J.Mora-Muscular activity during isometric incisal biting, Journal of Biomechanics,2014,47.

23. Min -Kyu Park, Sung-Min, Kyoung -In Yun, Je-Uk Park-Change in Bite Force and Electromyographic Activity of Masticatory Muscle in Accordance with Change of Occlusal Plane, Journal of Oral and Maxillofacial Surgery,2012,70:1960-1967.

24. Toru Takahashi, Fumiyo Hayakawa, Masanori Kumagai, Yoshinobu Akiyama, Kaoru Kohyama-Relations among mechanical properties, human bite parameters and ease of chewing of solid foods with various textures, Journal of food engineering,2009,95:400-409.

25. Lei Chen, Peter Alfred Proschel, Thomas Ricarrdo Morne burg-Influence of bite force on jaw muscle activity ratios in subject-controlled unilateral isometric biting,2010,20:961-966.

26. Veena jain, Vijay Prakash Mathur, Kumar Abhishek, Mohit Kothari-Effects of occlusal splint therapy on maximum bite force in individuals with moderate to severe attrition, Journal of Prosthodontic Research,2012,56:287-292.

27. Casey R.Lepley, Gaylord S.Throckmorton, Richard F.Ceen, Peter H.Buschang-Relative contributions of occlusion, maximum bite force and chewing cycle kinematics to masticatory performance, American Journal of Orthodontics and Dentofacial Orthopaedics, May 2011,139(5)606-613.

28. Mohannad M.Abdullah, Humam Saltaji, Hussein Abou-Hamed, Mohammed Youssef-The relationship between molar bite force and incisor inclination: A prospective cross-sectional study, International Orthodontics, 2014, 12:494-504.

29. Viviana Toro -Ibacache, Victor Zapata Munoz, Paul O’Higgins-The relationship between Skull morphology, Masticatory muscle force and cranial skeletal deformation during biting, Annals of Anatomy, 2016, 203:59-68.

30. D.Hellmann, T.Stein, W.Potthast, P.Rammelsberg, H.J.Schindler, S.Ringhof-The effect of force controlled biting on human posture control, Human Movement Science, 2015, 43:125-137.

31. Elham S, J.Abu Alhaja, Ibraheem A. Al Zoubi, Mohammed E. Al. Rousan, Mohammad M.Hammad-Maximum occlusal bite forces in Jordanian individuals with different dentofacial vertical skeletal patterns, European Journal of Orthodontics,2009:1-7.

32. A.Shimada, L.Baad-Hansen, P.Svensson-Effect of experimental jaw muscle pain on dynamic bite force during mastication, Archives of Oral Biology, 2015, 60:256-266. 
33. Rogerio Alexandre Modesto de Abreu, Max Domingues Pereira, Fabianne Furtado, Gabriela Pereira Ribeiro Prado, Wilson Mestriner Jr., Lydia Masako Ferreira- Masticatory efficiency and bite force in individuals with normal occlusion, Archives of Oral Biology,2014,59:1065-1074.

34. LIselotte Sonnesen, Peter Svensson-Jaw- motor effects of experimental jaw - muscle pain and stress in patients with deep bite and matched control subjects, Archives of Oral Biology,2013,58:1491-1497.

35. Demirhan Diracoglu, Kerem Alptekin, Ebru Demet Cifter, Burak Guclu, Ayse Karan, Cihan AksoyRelationship between maximal bite force and tooth wear in bruxist and non-bruxist individuals, Archives of Oral Biology,2011,56:1569-1575.

36. Veena Jain, Vijay Prakash Mathur, Abhishek kumar-A preliminary study to find a possible association between occlusal wear and maximum bite force in humans,2013,Acta Odontologica Scandinavica,71:93-101.

37. Yoshinori Hattori, Chiaki Satoh, Takeyasu Kunieda, Rui Endoh, Hisyuuki Hisamatsu, Makoto Watanabe-Bite forces and their resultants during forceful intercuspal clenching in humans, Journal of Biomechanics,2009,42:1533-1538.

38. Mortaza Bonakdarchian, Navid Askari, Masood Askari-Effect of face form on maximal molar bite force with natural dentition, Archives of Oral Biology,2009,54:201-204.

39. Marcelo Palinkas, Mariangela Salles Pereira Nassar, Flavia Argentato Cecilio, Selma Siessere, Marisa Semprini, Joao Paulo Machado -de-Sousa, Jaime Eduardo Cecilio Hallak, Simone Cecilio Hallak Regalo-Age and gender influence on maximal bite force and masticatory muscle thickness, Archives of Oral Biology,2010,55:797-802.

40. Duygu Koc, Arife Dogan, Bulent Bek, Meryem Yucel-Effects of increasing the jaw opening on the maximum bite force and electromyographic activities of jaw muscles, Journal of Dental Sciences,2012,7:14-19.

41. B.K.Biswas, S.Bag, S.Pal , Biomechanical analysis of normal and implanted tooth using bite force measurement,2012.

42. Sho Kayumi, Yoshiyuki Takayama, Atsuro Yokoyama, Nana Ueda, Effects of bite force in occlusal adjustment of dental implants on the distribution of occlusal pressure: comparision among three bite forces in occlusal adjustment, 2015 . 